ELEPHANT SEALS by Bob Evans, one of over 150 superb colour illustrations in Vanishing Species published by Time-Life (\$11.95)

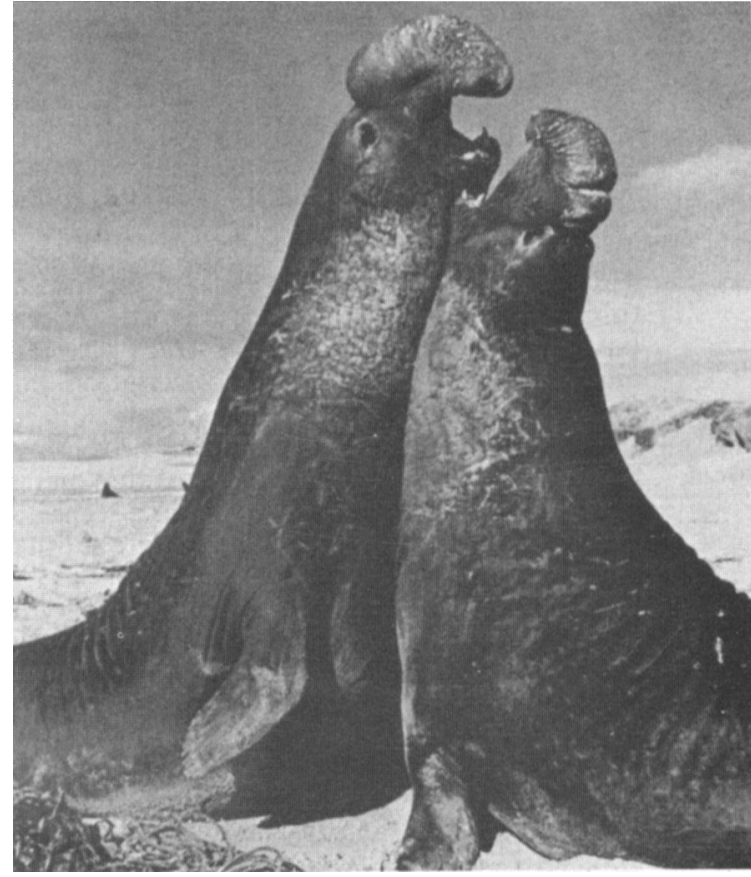

Occasionally one encounters a rather odd remark, such as the statement that the use of venom took hundreds of thousands of years to develop (some millions, surely!). On the whole, however, the information is sound, well presented and upto-date. There is a synoptic guide to identification, a good bibliography and a series of excellent colour photos.

Both these books will be significant additions to the library of any herpetologist, even if he is not specifically concerned with the African or North American faunas.

A. D'A BELLAIRS

\title{
All About Photographing Animals and Birds, by David Hodgson. Pelham Books, $£ 4.00$
}

Photographing wild animals is not easy. So many combinations of subject, setting and light conditions can occur and usually when you least expect them, that only years of experience can prepare one to cope with them. The author of this book obviously has this experience and also the knack of passing on his knowledge in a readable way. Every page is full of practical and useful hints, and the technique of taking the reader through different photographic situations and explaining why a particular lens, speed and aperture were used is the best that I have encountered.

The book covers a wide field from natural shots in the wild through to the very contrived studio portrait of the family dog. The comprehensive opening chapter on equipment should enable the would-be photographer with no previous knowledge to decide exactly what camera, lenses, and film are suitable for his particular subjects.

For the naturalist wanting to use photography as a means of recording or learning more about his particular subject there is an excellent section on making and using devices that will automatically photograph animals as they pass close to the camera. Night time flash photography and the use of extension tubes and bellows for extreme close up pictures are also covered in depth, and there is an extensive and up-to-date glossary of photographic terms. At first I was rather put off by the large number of photographs of family pets in anthropomorphic poses, but these do not seriously detract from the overall excellence of a well written book. 